\title{
NMDB database and global survey method
}

\author{
Petr Yu. Gololobov ${ }^{\circledR}$, Sergey A. Starodubtsev ${ }^{\circledR}$, Vladislav G. Grigoryev ${ }^{\circledR}$, Anton S. Zverev ${ }^{\circledR}$ \\ Correspondence \\ Yu.G. Shafer Institute of Cosmophysical Research and Aeronomy of SB RAS, Yakutsk, Russia, gpeter@ikfia.ysn.ru
}

\section{Keywords}

cosmic rays; neutron monitor; global survey method

\section{OPEN ACCESS}

This work is published under the Creative Commons Attribution 4.0 International license (CC BY 4.0) Please note that individual, appropriately marked parts of the work ma be excluded from the license may be excluded from the license other copyright conditions. other copyright conditions. If such third party material is not under the Creative Commons license any copying, editing or public reproduction is only permitted with the prior consent of the respective copy right owner or on the basis of relevant legal authorization regulations.

\begin{abstract}
The method of a global survey developed in the 1970s allows using a world-wide network of neutron monitor stations as a single multidirectional device. Wherein, receiving characteristics of each device, which reflects their geometries and geographical positions, are taken into account. Such an approach makes it possible to define the first two angular moments of the distribution function of cosmic rays in the interplanetary space at each hour of observation. With the creation in 2008/2009 and subsequent development of an international database of neutron monitors NMDB, for the first time it appeared an opportunity to use the global survey method in real-time mode. Such a situation creates a unique possibility to use the results not only for scientific researches but also for space weather forecasting. To use the data of the world-wide network of neutron monitors it is necessary to carry preliminary preparations. Thereby, in the current work, the main attention is attracted to a solution to some practical questions that arise when using the NMDB in real-time.
\end{abstract}

\section{Introduction}

Global Survey Method (GSM) (Altukhov et al. 1969) was developed in the late 1960s in the Yu.G. Shafer Institute of Cosmophysical Research and Aeronomy and allows using the entire world network of neutron monitor (NM) stations as a single multidirectional device oriented at each measured moment in different directions. This approach makes it possible to determine the dynamics of the angular distribution of cosmic rays (CRs) with a temporal resolution that depends on the capabilities of the network devices. The use of such methods makes it possible to carry out studies of various short-term processes of solar-terrestrial relationships and space weather using CR measurements.

Despite the long existence, more than half a century, of the method, the modernization of the GSM continues to this day. One of the important stages in the development of the GSM is associated with the creation of the international database NMDB in 2007. This database made it possible to implement the GSM in real-time, which meant the application of the method for monitoring and forecasting space weather. Subsequent studies in this direction are presented in the works by Grigoryev et al. (2015, 2017, 2019). This paper presents the current state of the method and focuses on some practical issues of the implementation of the GSM based on NMDB data.

\section{Method}

\subsection{Global survey method}

GSM is a variant of the spherical analysis of experimental NM data and is based on so-called receiving vectors (Krymsky et al. 1981). The angular distribution of CR, when represented as a series 
of spherical functions, will be described by the multidimensional vector $\vec{A}=\left(a_{n}^{m}, b_{n}^{m}\right)$, where $a_{n}^{m}$ and $b_{n}^{m}$ are the decomposition coefficients, and $n \geq m \geq 0$. Determining the corresponding receiving vectors $\overrightarrow{z_{n}^{m, j}}=\left(x_{n}^{m, j}, y_{n}^{m, j}\right)$, taking into account the relationship between the primary CR distribution and the intensity measured by ground-based detectors $I^{j}$, we have:

$$
I^{j}=\sum_{n=0}^{\infty} \sum_{m=0}^{n}\left(a_{n}^{m} x_{n}^{m, j}+b_{n}^{m} y_{n}^{m, j}\right)
$$

If there is a sufficient number of NM stations, it is possible to construct a system of independent equations, the solution of which will make it possible to determine the required angular distribution of CR $\vec{A}$ from the NM measurements. If it is required to determine the first spherical harmonic (diurnal anisotropy), it is necessary to have data from at least 4 evenly spaced NM stations, and for the second spherical harmonic (semidiurnal anisotropy) - 9.

Since the used NM stations have different energy sensitivities, it is necessary to introduce normalizing factors that bring the instrument readings to a certain nominal one:

$$
k_{n}^{j}=\frac{\int_{R_{c}^{j}}^{\infty} W^{j}(E) f_{n}(E) d E}{\int_{R_{c}^{j}}^{\infty} W^{0}(E) f_{n}(E) d E},
$$

where $E$ is the particle energy, $W^{j}(E)$ is the coupling coefficient (Dorman 1957) of the j-th NM station and $f_{n}(E)$ is the assumed energy spectrum of the $n$-th order variations.

Based on the above the equation (1) becomes:

$$
I^{j}=\sum_{n=0}^{\infty} \sum_{m=0}^{n}\left(a_{n}^{m} x_{n}^{m, j}+b_{n}^{m} y_{n}^{m, j}\right) k_{n}^{j}
$$

Reducing equation (3) to matrix form, we obtain:

$$
I=M A,
$$

where $M$ is the matrix of receiving vectors, and $I$ and $A$ are vectors of the observational data and the expected CR distribution, respectively. The solution to equation (4) has the form:

$$
I=\left(M^{T} M\right)^{-1} M^{T} I
$$

The resulting system of equations is solved by the least-squares method under the assumption that the series is rapidly decaying.

In practice, the CR intensity included in equation (3) observed by the detector may contain oscillations that are not associated with variations in the primary intensity, for example, low-frequency instrumental oscillations. Therefore, when solving (3), the high-frequency component of the intensity $I_{h . f}$ is used, which is found from the equation

$$
I_{h . f .}(t)=I(t)-\sum_{t^{\prime}=t+T}^{t+T} \frac{I\left(t^{\prime}\right)}{(2 T+1)},
$$

where $I(t)$ is the observed intensity, and $T$ is the number of points of moving average, which can be taken equal to 18 hours. 


\subsection{Using NMDB data in the global survey method}

The database of neutron monitors NMDB (https://www.nmdb.eu/nest/help.php\#helpstations, last accessed April 8, 2021) was created in 2007. The value of such a database lies, first of all, in the presentation of data from the global NM network in real-time, as well as the provision of high temporal resolution data in a unified form. Over 50 stations are presented in NMDB, which are distributed throughout the planet (figure 1). The description of the database, as well as the areas of scientific problems in which it is applied, are presented in the work (Mavromichalaki et al. 2011).

It is important to note that the effective CR energies recorded by the NMDB can be roughly estimated as $10-25 \mathrm{GeV}$.

Implementation of the GSM using the NMDB data allows tracking the dynamics of the CR angular distribution in real-time. However, this may pose some obstacles. First, the selection of the high-frequency component by equation (6) loses its meaning due to the absence of subsequent points; secondly, the often occurring registration errors (hardware failures) at various NM stations can significantly affect the result of the implementation of the GSM. Therefore, to take into account these problems, the following methods of processing primary data and analyzing the final results are used. In contrast to equation (6), the primary filtering method is replaced by using a linear trend for the last 24 hours, which is determined by the least-squares method. In this case, $I_{h . f}(t)$ will be determined as the difference between the observed variations and the trend. Errors in the registration of individual stations of the NM network can be taken into account by analyzing the output data of the GSM. Comparing the obtained solutions of the system (5) and the primary equation (4), it is possible, using Chauvenet's criterion (Taylor 1985), to exclude those NM stations whose data are more divergent from the rest.

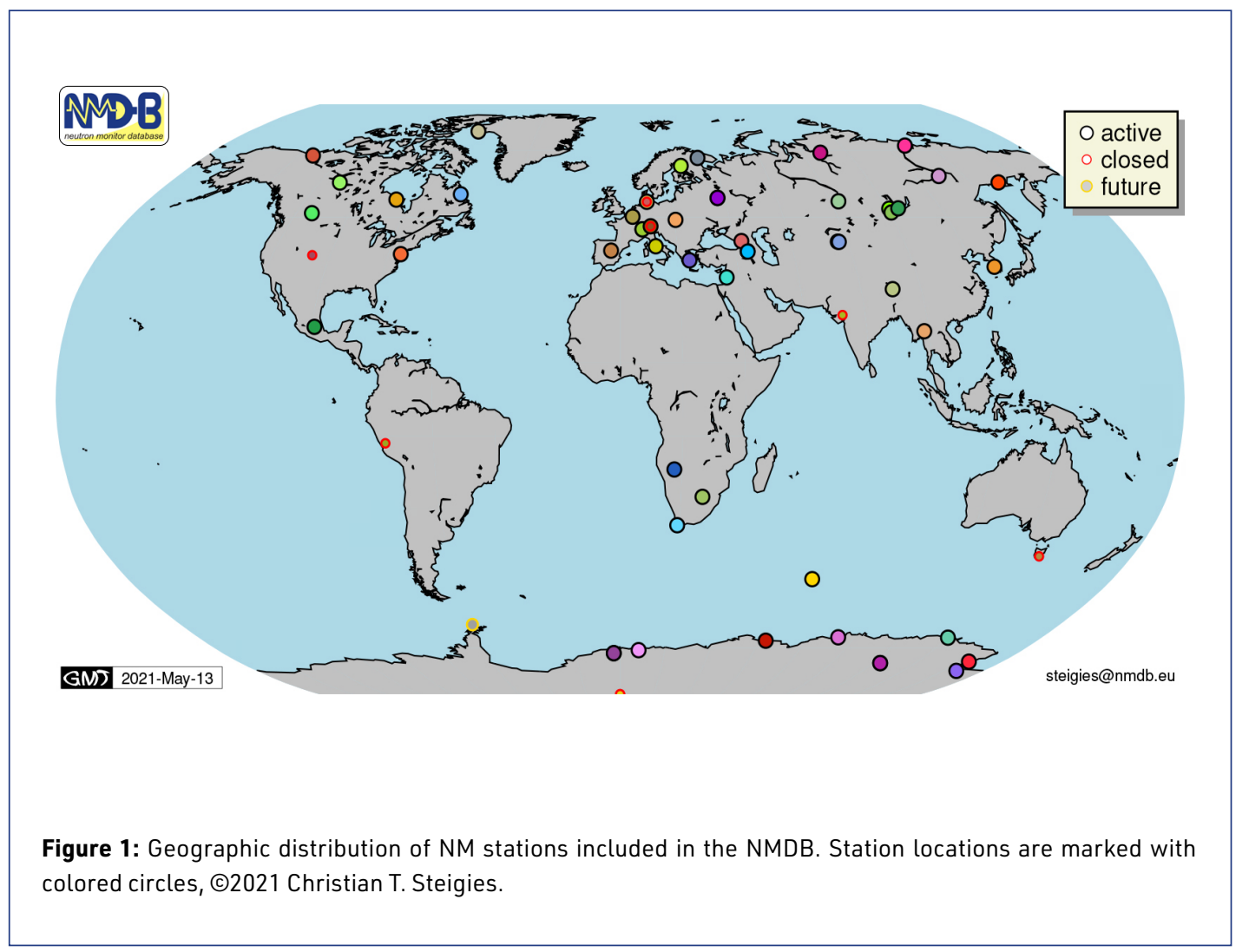




\subsection{Forecasting geomagnetic storms}

The creation of the NMDB network in 2007 served as an impetus for the implementation of the GSM method in real-time and in the study of the possibilities of the practical application of its results. The work carried out over the past 10 years in this direction (Grigoryev et al. 2011a, 2015, 2017, 2019; Starodubtsev et al. 2019) showed that it is possible to predict geomagnetic storms. As it turned out, the parameters of the angular distribution of CRs, identified using the GSM, actively respond to the approach of geoeffective disturbances of the interplanetary medium to the Earth. Analysis of the results of the GSM for 2012-2018 made it possible to establish that anomalous changes in the magnitude and direction of the first harmonic vector $\left(a_{11}\right.$ and $\left.b_{11}\right)$ and the amplitudes of the zonal (north-south) components $\left(a_{00}, a_{10}\right.$ and $\left.a_{20}\right)$ of the CR distribution can serve as predictors of geomagnetic disturbances. For the zonal components, the critical values of individual positive values or their sums were determined, the excess of which, with a probability of $75 \%$, is a precursor of a sharp decrease of Dst-index at the beginning of the geomagnetic storm (Grigoryev et al. 2019).

The results of the implementation of the GSM according to the NMDB data are presented online in real-time at the link http://www.ysn.ru/ starodub/SpaceWeather/global_survey_real_time. html (last accessed April 8, 2021). Here, the behavior of the main parameters of the CR distribution for the last 72 hours is presented: isotropic intensity $I$, the modulus of the harmonic vector and the sums of positive and negative values of the zonal harmonics, as well as the values of the components and vectors of the first and second harmonics in the GSE coordinate system.

\section{Conclusions}

A brief description of the implementation, based on data from the world network NMDB, of the GSM in real-time is given. Based on the analysis of the obtained parameters of the CR angular distribution, the state of the interplanetary medium is monitored and geomagnetic disturbances are predicted. Monitoring results in real-time are displayed on the internet through the Web link http://www.ysn. $\mathrm{ru} /$ s starodub/SpaceWeather/global_survey_real_time.html (last accessed April 8, 2021).

\section{Acknowledgments}

The work is carried out with partial support of the ShICRA Basic Research Program no. AAAAA17-117021450058-6 and the RFBR grants Nos. 18-42-140002-r_a.

We acknowledge the NMDB database (https://www.nmdb.eu/), founded under the European Union's FP7 programme (contract no. 213007) for providing data.

\section{References}

Altukhov, A. M., Krymsky, G. F., Kuzmin, A. I., 1969, Acta Physica, 29, 457

Dorman, L. I., 1957, Variatsii kosmicheskikh luchei [Cosmic Ray Variations], Gostekhizdat Publ. (in Russian)

Grigoryev, V. G., Starodubtsev, S. A., 2011a, Proc. 32nd ICRC, 11, 99, D0I: https://dx.doi.org/10.7529/ICRC2011/V11/0359

Grigoryev, V. G., Starodubtsev, S. A., Krymsky, G. F., et al., 2011b, Proc. 32nd ICRC, 11, 9, https://galprop.stanford.edu/elibrary/icrc/2011/ papers/SH3.6/icrc0360.pdf (last accessed May 20, 2021)

Grigoryev, V. G., Starodubtsev, S. A., 2015, Bull. of the RAS: Physics, 79, 649, DOI: https://dx.doi.org/10.3103/S1062873815050226

Grigoryev, V. G., Starodubtsev, S. A., Gololobov, P. Yu., 2017, Bull. of the RAS: Physics, 81, 200, D0I: https://dx.doi.org/10.3103/S10 62873817020198

Grigoryev, V. G., Starodubtsev, S. A., Gololobov, P. Yu., 2019, Solar-Terrestrial Physics, 5, 93, DOI: https://dx.doi.org/10.12737/stp-53201911 Krymsky G. F., Kuzmin, A. I., Krivoshapkin, P. A., et al., 1981, Kosmicheskie luchi i solnechniy veter [Cosmic rays and solar wind], ed. Shafer, G.V., Nauka Publ. (in Russian)

Mavromichalaki, H., Papaioannou, P., Plainaki, C., 2011, Advances in Space Research, 47, 2210, D0I: https://dx.doi.org/10.1016/j. asr.2010.02.019 
Starodubtsev, S. A, Grigoryev, V. G., Gololobov, P. Yu., 2019, Journal of Physics: Conference Series, 1181, 012011, D0I: https://dx.doi. org/10.1088/1742-6596/1181/1/012011

Taylor, J. R., 1982, An Introduction to Error Analysis: Study of Uncertainties in Physical Measurements, ed. McGuire, A., University Science Books Publ.

\section{Questions and answers}

Rolf Bütikofer: Do you issue spwx alarms by SMS or e-mail ? Who are the stakeholders of your application? Answer: The only technique of alert is the link https://www.ysn.ru/ starodub/SpaceWeather/global_survey_real_time.html (last accessed April 8, 2021).

Hazel Bain: Does the method forecast the dst value?

Answer: The method predicts the geomagnetic storms with Dst $<-50 \mathrm{nT}$.

Monica Laurenza: What is the false alarm rate?

Answer: Statistical analysis of the results shows that the effectiveness of forecasting $~ 75 \%$. It strongly depends on the critical values that are selected.

Christian T. Steigies: How do you access real-time data from NMDB? Via NEST or the real-time files?

Answer: The real-time files.

Christopher Light: Are you predicting that a geomagnetic storm will occur? Or are you predicting the magnitude of the dst disturbance?

Answer: Yes, we predict that a geomagnetic storm will occur. And Dst<-50 nT.

Eugenia Eroshenko: What do you do with occasional spike or drift in the stations if it occurs?

Answer: They can be eliminated using Chauvinet's criterion (see the text for details)

John Clem: How is the accuracy of your SW predictions dependent on the global NM viewing resolution? Answer: The accuracy is highly dependent on the distribution of stations. This especially applies to the station collecting CRs from the regions of the Pacific Ocean and Earth's poles. 